\title{
The effect of haptic guidance, aging, and initial skill level on motor learning of a steering task
}

\author{
Laura Marchal-Crespo • Stephanie McHughen • \\ Steven C. Cramer · David J. Reinkensmeyer
}

Received: 3 April 2009/ Accepted: 18 September 2009/Published online: 10 October 2009

(C) The Author(s) 2009. This article is published with open access at Springerlink.com

\begin{abstract}
In a previous study, we found that haptic guidance from a robotic steering wheel can improve shortterm learning of steering of a simulated vehicle, in contrast to several studies of other tasks that had found that the guidance either impairs or does not aid motor learning. In this study, we examined whether haptic guidance-as-needed can improve long-term retention (across 1 week) of the steering task, with age and initial skill level as independent variables. Training with guidance-as-needed allowed all participants to learn to steer without experiencing large errors. For young participants (age 18-30), training with guidance-as-needed produced better long-term retention of driving skill than did training without guidance. For older participants (age 65-92), training with guidance-as-needed improved long-term retention in tracking error, but not significantly. However, for a subset of less skilled, older subjects, training with guidance-as-needed significantly improved long-term retention. The benefits of guidancebased training were most evident as an improved ability to straighten the vehicle direction when coming out of turns. In general, older participants not only systematically performed worse at the task than younger subjects (errors $\sim 3$
\end{abstract}

L. Marchal-Crespo ( $₫)$

Department of Mechanical and Aerospace Engineering,

Henry Samueli School of Engineering,

4200 Engineering Gateway Building, Irvine,

CA 92697-3975, USA

e-mail: 1marchal@uci.edu

S. McHughen · S. C. Cramer

Departments of Anatomy and Neurobiology,

University of California, Irvine, USA

D. J. Reinkensmeyer

Departments of Mechanical and Aerospace Engineering and

Biomedical Engineering, University of California, Irvine, USA times greater), but also apparently learned more slowly, forgetting a greater percentage of the learned task during the 1 week layoffs between the experimental sessions. This study demonstrates that training with haptic guidance can benefit long-term retention of a driving skill for young and for some old drivers. Training with haptic guidance is more useful for people with less initial skill.

Keywords Haptic perception - Motor control · Motor learning · Timing

\section{Introduction}

Haptic guidance is a motor-training strategy in which a human or machine trainer physically interacts with the participant's limbs during movement training, guiding them through a desired movement (Feygin et al. 2002; Hagman 1983; O’Malley et al. 2006; Winstein et al. 1994). This strategy is commonly used to reduce performance errors for tasks that are dangerous to practice, such as learning to walk after a neurologic injury or learning complex gymnastics moves like flips. Besides providing a safety benefit, a common concept is that physically demonstrating a movement may help people learn how to perform it. However, even though haptic guidance is often used in motor training, there is currently little evidence that robotic guidance is beneficial for human motor learning beyond enhancing safety, compared with unassisted practice. In fact, a long-standing hypothesis in motor learning research is the guidance hypothesis, which states that providing too much guidance during training of movement will impair motor learning because it changes the inputoutput relationship of the task to be learned, and, therefore, obviates the motor system from learning to deal with the 
actual relationship (Salmoni et al. 1984; Schmidt and Bjork 1992). A number of studies have confirmed this hypothesis finding that physically guiding movements does not aid in motor learning and may in fact hamper it (Hagman 1983; Winstein et al. 1994; Armstrong 1970; Tsutsui and Imanaka 2003; Gillespie et al. 1998; O'Malley et al. 2006; Wallis and Koh 2002; Feygin et al. 2002; Liu et al. 2006; Kahn et al. 2006).

We recently conducted an experiment the results of which contradicted the guidance hypothesis (MarchalCrespo and Reinkensmeyer 2008b). We found that young unimpaired participants who practiced learning to drive a simulated wheelchair with compliant guidance from a robotic steering wheel learned better than participants who practiced without physical guidance. Participants who received guidance reduced their error by initiating their turns earlier, following the example provided by the robotic guidance. In other words, they learned to time their movements better, apparently because they were positively influenced by the timing example provided by the robotic steering wheel. These results show that a refinement of the simplistic interpretation of the guidance hypothesis, that guidance categorically impairs learning, is needed, a refinement that describes with greater detail under what task conditions, and for what kind of participants, guidance may have benefits.

The goal of the present study was to extend the original steering study in three ways. First, in the prior study, the performance improvements observed in the initial study were measured immediately after the training, and thus it was unclear if they persisted over a longer time scale. There is a body of work on haptic guidance that have showed the benefits of human-machine haptic interactions on driving performance (Suzuki and Jansson 2003; Griffths and Gillespie 2005; Jones and Sarter 2008). However, there is little evidence that the benefits of such haptic guidance persist once the haptic guidance is removed. One goal of the present study was, therefore, to determine if the guidance-related learning enhancement persists at a long-term (1 week later) retention test.
Second, we sought to determine the effect of age on the benefits of guidance. If guidance can improve motor learning, it is important to understand the conditions under which it is beneficial, and to develop applications that exploit the beneficial features of guidance for populations who have difficulty learning. Motor performance and motor learning have been documented to be impaired with aging (Dijk et al. 2007; Carnahan et al. 1996; Wishart and Lee 1997; Swanson and Lee 1992; Swinnen 1998; Wishart et al. 2002; Voelcker-Rehage 2008), even though the need to learn new skills remains crucial with aging (e.g. learning to drive a new car or a powered wheelchair, Hedel and Dietz 2004). Haptic guidance might provide a means to improve motor learning in older people.

Third, we sought to determine the effect of initial skill level on the benefits of training with guidance. An influential motor learning theory, the challenge point theory (Guadagnoli and Lee 2004) states that optimal learning is achieved when the difficulty of the task is appropriate for the individual participant's level of expertise (i.e. when the challenge point is reached). Haptic guidance makes a task easier, and thus might be predicted to have greater benefit for initially less skilled trainees.

\section{Methods}

Steering simulator and guidance controller

We used the steering simulator and guidance control strategy introduced in (Marchal-Crespo and Reinkensmeyer 2008b) to perform the experiments. The driving simulator consists of a 3D graphical presentation of a circuit defined by a black line on a floor that participants are required to follow. The virtual circuit is $120-\mathrm{m}$ long, has six curves, and it takes approximately $60 \mathrm{~s}$ to drive through the circuit. Participants steer a virtual power wheelchair through the circuit from the point of view of sitting on the wheelchair, using a conventional game force feedback steering wheel (Fig. 1, Logitech MOMO॰ 100\$). Vehicle
Fig. 1 Left participant driving the wheelchair simulator. Right top view of the pathway the participants were required to complete
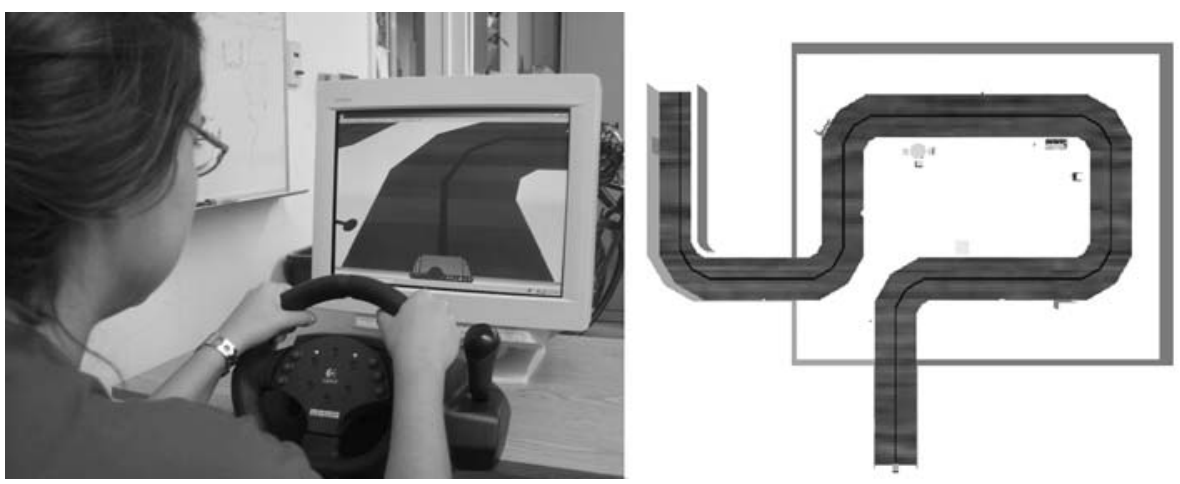
steering becomes more difficult as the vehicle speed increases (Zhai et al. 2004). In this study, to eliminate this possibly confounding variable, we programmed the chair to move at a constant, relatively fast speed for the entire task ( $2 \mathrm{~m} / \mathrm{s})$.

As explained in Marchal-Crespo and Reinkensmeyer (2008b), we developed a guidance control algorithm such that the steering wheel applied forces to the participant's hands that corrected the steering wheel motion to bring the virtual wheelchair back to the desired circuit, when the participant created steering errors. The guidance is defined as the force that the haptic steering wheel applies to the driver's hands. The guidance control algorithm is designed to assist only as needed, i.e. guidance is not provided if the error is zero, and the guidance level (the impedance of the guiding robot) is decreased as the driver learns how to steer without making large errors, but increases with error. In this way, as the guidance is reduced, the driver is free to drive with larger errors, but there is still a larger force for larger errors making sure the vehicle does not deviate too far from the desired path.

The dynamics of the virtual power wheelchair are nonholomonic, i.e. the vehicle cannot change direction instantaneously (Marchal-Crespo and Reinkensmeyer 2008b). Thus, when turning in curves the driver has to start the movement before the track changes direction to minimize the tracking error. The driving action is then dependent on what the driver sees in front of him instead of the nearest point from the wheelchair to the black line. Likewise, the guidance controller uses the look-ahead distance error $\left(e_{\mathrm{dis}}\right)$, look-ahead direction error $\left(e_{\mathrm{ang}}\right)$, and the derivative of the wheelchair orientation angle $\left(e_{\text {ang }}\right)$ to calculate the required assistive force. The optimal lookahead distance used for the experiments described below was chosen by trial and error and had a value of $0.84 \mathrm{~m}$. With this optimal look-ahead distance, the guidance algorithm moved the steering wheel in such a way that the circuit-tracking mean error was very small $(0.011 \mathrm{~m})$ when no driver held the steering wheel. The guidance force was defined by

$F_{\text {assist }}=K_{d} \cdot e_{\mathrm{dis}}+K_{a} \cdot e_{\mathrm{ang}}+B_{a} \cdot \dot{e}_{\mathrm{ang}}$

Thus, as the error becomes larger, the guidance force $F_{\text {assist }}$ becomes larger (range 0-2 Nm), with control gains $K_{d}, K_{a}$, and $B_{a}$ (with initial values $16 \mathrm{~N}, 16 \mathrm{Nm}$ and $3 \mathrm{Nm}^{*} \mathrm{~s}$, respectively). We adapted the control gains (i.e. firmness of guidance) using an adaptive algorithm described in Emken et al. (2007):

$G_{i+1}=f_{\mathrm{R}} \cdot G_{i}+g_{\mathrm{R}} \cdot\left|x_{i}-x_{d}\right|$

where $G$ represents the value of a control gain in the guidance controller, $f_{\mathrm{R}}$ is the robot forgetting factor $\left(f_{\mathrm{R}}\right.$ $=0.099986), g_{\mathrm{R}}$ is the robot learning gain $\left(g_{\mathrm{R}}=0.002\right.$ when adapting $K_{d}$ and $K_{a}$, and $g_{\mathrm{R}}=0.0002$ for $B_{a}$ ), $x_{i}$ is the position or angle in the $i$ time-step iteration and $x_{d}$ is the desired position or direction defined by the path. Note that the guidance algorithm adapts the control gain value based on the driving error and the previous control gain value. Note also that $f_{\mathrm{R}}$ must be less than one and greater than zero to systematically decrease the value of the guidance when the path tracking error $\left(x_{i}-x_{d}\right)$ is small. For more information regarding the steering simulator and guidance controller, we refer the reader to Marchal-Crespo and Reinkensmeyer (2008b).

\section{Experimental protocol}

All experiments were approved by the Institutional Review Board of the University of California at Irvine, and participants provided informed consent. Sixty-two, healthy, right-handed adult participants consented to take part in the experiment. Participants were assigned in two groups depending on their age. Thirty participants were assigned in the "young" group (age $24 \pm 3.24$ ). Thirty-two participants were assigned into the "old" group (age $78.13 \pm$ 7.52). Participants in the "young" group were randomly assigned in two groups: the "Guidance-as-needed" group and the "No guidance" group. The initial half of older subjects were randomly assigned to either training with guidance-as-needed or no guidance on the second training day. However, at this point, we noticed that initial driving errors differed between groups. Because we wanted to evaluate the effect of initial skill level on the benefits of training with guidance, an imbalance in initial driving errors could have confounded this comparison. To prevent an imbalance, therefore, the remainder of older subjects was assigned to one of the two groups using adaptive randomization methods that insured comparable levels of error across the two groups. At the end of this procedure, 17 elderly participants ended in the guidance group, and 14 in the no guidance group.

All participants participated in three experimental sessions on three different days. On the first day, all participants completed the same driving circuit 15 times, without robotic guidance from the steering wheel. All participants then returned 1 week later for a second experimental session. In this session, they first drove the same circuit two times without guidance to test the retention from the training of the previous week. After $5 \mathrm{~min}$, participants in the no guidance group drove the simulated wheelchair through the circuit 20 times without robotic guidance. Participants in the guidance-as-needed group drove 15 times with haptic guidance from the steering wheel, and five times without guidance. All participants returned a week later for a third experimental session to test long-term retention, and on this day they drove through the circuit 
two more times. The time required to drive the 120-m long circuit was approximately $60 \mathrm{~s}$. Participants paused for $10 \mathrm{~s}$ between the trials.

All participants were informed to drive after the black line that was displayed down the center of the screen with the smallest error possible. None of the participants had prior experience with the simulation program or the steering wheel. Participants in the no guidance group did not receive any guidance during the experiment. Participants in the guidance-as-needed group received adaptive guidance that was adjusted according to Eq. 2 during 15 trials on the second day. They were instructed to follow along lightly with their arms during the first trials, and to try to continue to cooperate with the guidance force as it was reduced. They were told that the guidance would be removed after 15 trials.

\section{Data and statistical analysis}

For each trial, the tracking error, defined as the mean of the absolute value between the center of the simulated wheelchair and the black line, was measured. The data from one participant in the older, guidance-as-needed group was discarded because she did not follow the instructions correctly despite the coaching, instead appearing to remain passive throughout the experiment, increasing the errors with time, instead of reducing them, as did the rest of participants. Some participants in the older group were not able to finish some trials, because they got lost and could not find the way back to the line, or because they created a large error and crashed into the simulated walls. Specifically, for the older participants for trial 1, 13 participants did not finish, and likewise, using the notation (trial number: number of subjects who did not complete) - 2:11, 3:4, 4:4, 5:5, 6:5, 7:4, 8:2, 9:2, 10:1, $11: 2,12: 2,13: 3,14: 1,15: 4,15: 3,17,18,21,22,32,34$, $36: 1,38: 2$. In the younger participants group, only one participant could not finish trial 28, and 2 participants did not finish trial 32. Data from these participants on these trials were excluded from the analysis, as the task nature changed completely on these trials, requiring participants to perform a searching behavior once they were lost.

To determine whether the guidance reduced the tracking error compared with no guidance, we performed a repeated measures one-way analysis of variance (ANOVA) across the relevant trials. The initial steering skill level was calculated as the mean between the tracking error created at the end on day 1 (trial 15), and trial 17. To test the correlation between error reduction and initial skill level, we performed a Pearson's correlation test in the guidance and no guidance groups. We followed the method described in Zar (1984) to compare the resulting two regression lines slopes. For calculating retention, data from one subject was discarded, because the mean tracking error at trial 15 was higher than error on trial 1 , but lower than trial 16 .

To understand what skill components of making turns changed with training, we analyzed different segments of the turns. The circuit's curves were defined by two straight lines of 2-m long such that in a curve there are a total of three changes of line direction of $30^{\circ}$ each. We defined the first curve's half as the segment from 1-m ahead of the first curve's change of direction and the second direction change (i.e. when the curve straightened). We defined the second curve's part as the segment from the second curve's change of direction and $1 \mathrm{~m}$ after the third direction change. We defined straight lines as the segments that are not first or second halves of curves. We also tested if there were differences between groups in the turn-initiation distance before curves, and the turn-rectification distance after curves. We defined the turn-initiation distance as the distance ahead the curve at which participants reached $60 \%$ of the maximum turning speed before the track changed direction. We defined the turn-rectification distance as the distance reached when the participants reduced their turning speed to $40 \%$ of the maximum turning speed after the last curve's direction change. The increase in turn-initiation distance from trials 17 to 39 in both assistance strategies in the young group did not follow a normal distribution (Shapiro-Wilk test of normality $P=0.036$ and $P=0.037$, respectively). To test differences between guidance groups in learning the turn initiation in the young group we performed a Mann-Whitney test, whereas we performed a parametric $t$ test in the elderly group. To test relation between turn-initiation distance and turn-rectification distance with mean error, we performed a Pearson's correlation test using data only from last day (trials 38 and 39).

\section{Results}

Participants learned the task during an initial training session, with older subjects exhibiting larger errors

All participants first learned the steering task in a training session without any haptic guidance (Fig. 2). Performance gradually improved over the 15 training trials of this training session, leveling to a near constant value, for both old and young participants. However, older participants performed systematically worse than younger participants (Fig. 3, $P<0.012$ ), with a mean error that was about $3 \times$ larger in all stages of training. Figure 4 compares example trajectories created by younger and older participants at trial 15. Large oscillations exhibited by older participants when leaving the curves contributed to poorer performance when following straight lines. 
Fig. 2 Tracking errors during the three training and testing days. a Young subjects on day 1. Note that neither the assistance or no assistance group received guidance on day 1. b Young subjects on days 2 and 3 . The assistance group received gradually reducing assistance from trials 18 to 31 . Trials 38 and 39 were a retention test without assistance on day 3. c Old subjects on day 1 d Older subjects on days 2 and 3. Error bars in all plots show $\pm 1 \mathrm{SD}$

Fig. 3 Tracking errors during the three training and testing days. a Comparison of tracking error between old and young subjects in the no assistance group, across all 3 days. b Comparison of tracking error between old and young subjects in the assistance group, across all 3 days. Error bars in all plots show \pm 1 SD

Fig. 4 a Trajectories followed for each of the young participants at the end of training day 1 (trial 15). b Trajectories performed by each of the old participants in trial 15. Old participants created significantly larger tracking errors than young participants, and showed large oscillations when leaving the curves producing poor performance during straight lines
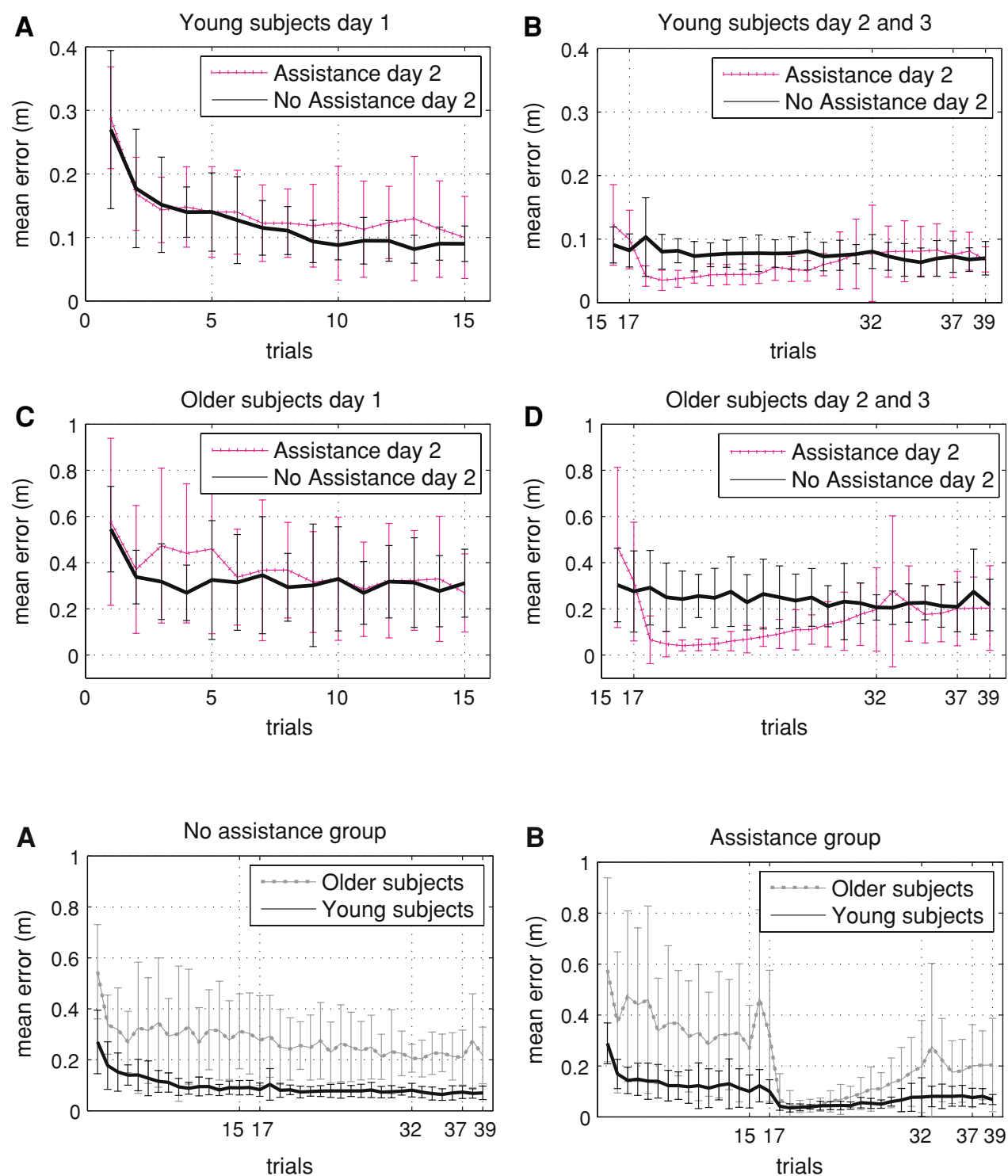

A Path followed by each young subject in trial 15

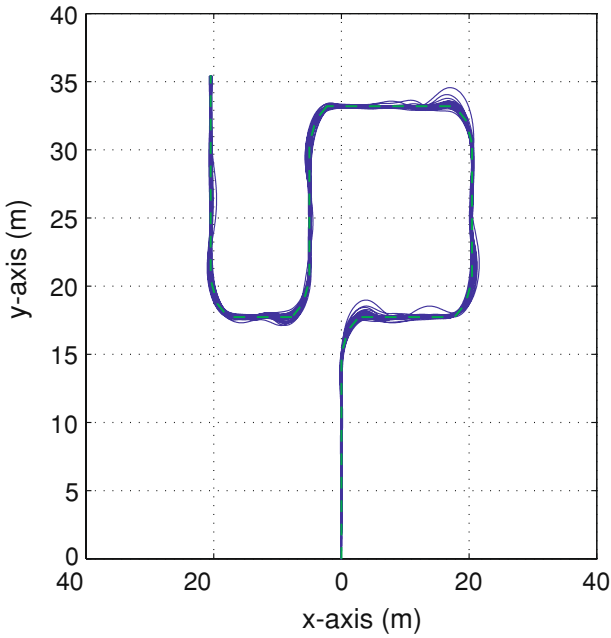


Guidance significantly reduced tracking error when it was applied during training

After 1 week of the initial training session, we then measured how well the participants learned to steer the virtual power wheelchair through the simulated environment after they trained with guidance-as-needed, or no guidance from a robotic steering wheel (Fig. 2). The robotic assistance provided by the steering wheel reduced steering errors for both younger and older participants when it was applied. For example, guidance-as-needed reduced the tracking error on the first trial when guidance was applied, compared with the last trial without guidance, in both the young participants' group (Fig. 2b, ANOVA, $P<0.001$ ), and old participants' group (Fig. 2d, ANOVA, $P<0.001$ ), and resulted in better steering performance across the trials it was applied (trials $18-28$ for young participants, $P<0.01$, and trials 18-29 for old participants, $P<0.03$ ). Old subjects performed at the same level as young subjects during the first trials when guidance was applied (trials 18-24), but performed significantly worse in all other trials (Fig. 3b, $P<0.05$ ). Because the guidance was reduced gradually based on the subject performance, when the robotic guidance was removed in trial 33 , there was not a significant increment in error when compared with the last trial without guidance in both, young, and old participants.

Training with guidance significantly improved longterm retention of the task, but just for younger subjects

In the young participants' group, the guidance-as-needed group showed better long-term learning characterized by a significant reduction in the mean tracking error from trial 17 to long-term retention, 1 week later (trial 39) (Fig. 5a, one-tailed $t$ test, $P=0.042$ ). However, this benefit of guidance-based training was not detected for the older participants. Specifically, despite the fact that the group of older subjects who received guidance-as-needed showed a larger tracking error reduction, the improvement was not significant (Fig. 5a, one-tailed $t$ test, $P=0.16$ ).

The training benefits of haptic guidance were not age dependent

Young participants significantly benefited more from training with guidance-as-needed than training without guidance, whereas older participants, taken as a group, showed only a tendency of greater benefits from guidance. However, we did not find significant differences between age groups in the proportion of error reduction (i.e. amount of error reduction from trial 17 to trial 39, over initial error in trial 17) in the guidance group, or the no guidance group (Fig. 5b, assistance group $P=0.649$, no assistance group, $P=0.796)$. Thus, the training benefits of haptic guidance did not appear to be age dependent, and, instead, the high intersubject variability of older participants may have accounted for the lack of a significant training benefit of guidance, when the group was taken as a whole.

Training with guidance significantly improved longterm retention more for initially less skilled subjects

We investigated the effect of the initial steering skill level (i.e. the steering error before training with guidance) on the effectiveness of haptic guidance, and found a significant tendency of better long-term retention for less skilled participants (Fig. 6a). Specifically, we found that there was a significant linear relationship between initial skill level and

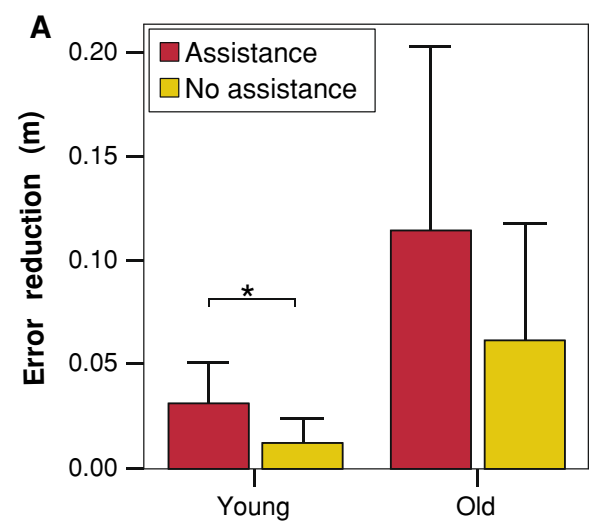

Fig. 5 a Long-term reduction in steering performance with and without haptic training. The plots compare the change in steering performance from trials 17 to 39 . Trial 17 was the last initial practice trial on Day 2 before training with guidance. Trial 39 was the final retention test trial on Day 3, 1 week later. b Proportional long-term

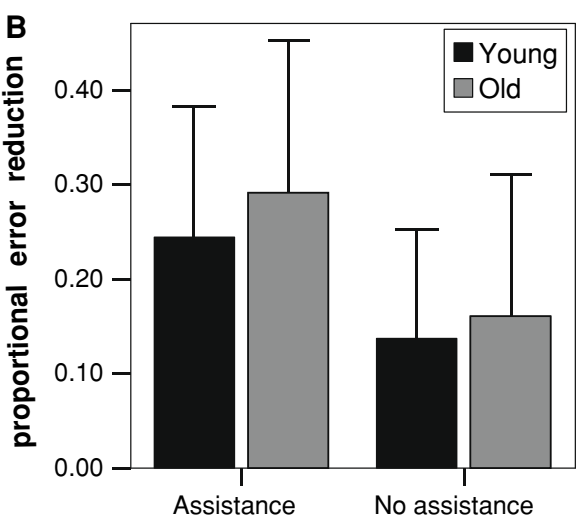

reduction in steering performance with and without haptic training for the young and old groups. The proportion of error reduction was calculated as the change in steering performance from trials 17 to 39 over performance in trial 17 
Fig. 6 Effect of initial skill level on the benefits of training with haptic guidance. All plots compare the change in steering performance from trials 17 to 39. Trial 17 was the last initial practice trial on Day 2 before training with guidance. Trial 39 was the final retention test trial on Day 3, 1 week later. a Effect of haptic guidance based on initial skill level for all participants. The guidancetrained group showed a significantly greater long-term error reduction for initially less skilled participants, when compared with the error reduction for the no guidance group. b Long-term reduction in steering performance in younger subjects with and without haptic training. c Longterm reduction in steering performance in older subjects with and without haptic training
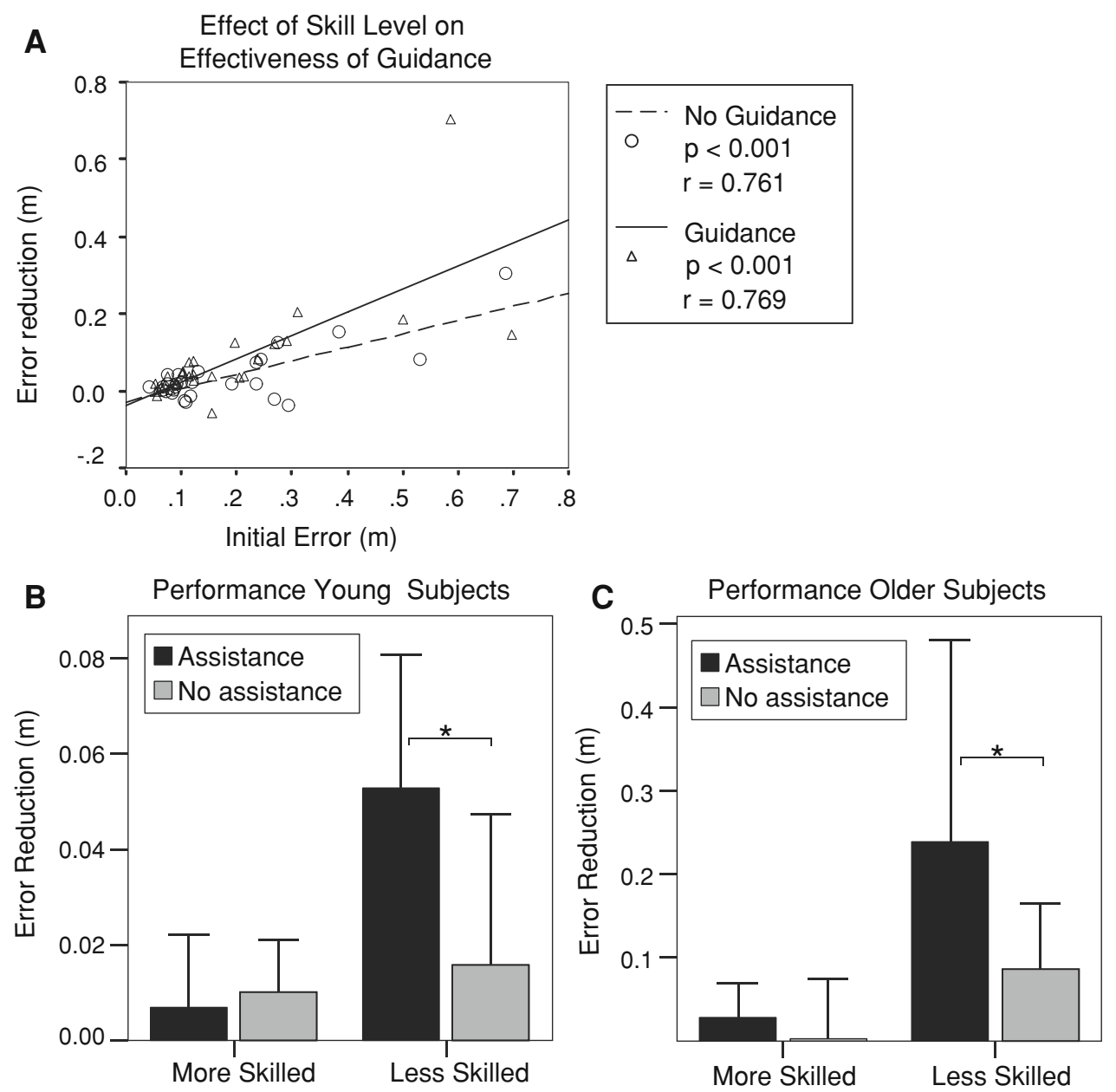

error at retention, for both guidance-trained (Pearson's correlation $r=0.721, P<0.001)$, and non-guidance-trained groups (Pearson's correlation, $r=0.769, P<0.001$ ). When we compared the slope of the two regression lines for the two groups, we found a significant greater slope for the guidance group (one-tailed $t$ test, $P=0.03$ ) suggesting that training with haptic guidance benefited the initially less skilled participants more than it did the more proficient participants.

Based on this finding, we analyzed if the guidance-asneeded was indeed more beneficial than no guidance for a subset of less skilled older subjects. We divided the older group into two different subsets based on their initial skill level. Older participants that performed systematically worse on trials 15 and 17 (mean performance error $<0.225$ $\mathrm{m})$ were assigned into the less skilled group $(n=15)$, whereas the rest were assigned into the more skilled group ( $n=11)$. In the older less skilled participants' group, the guidance-as-needed group showed better long-term learning (Fig. 6c, one-tailed $t$ test, $P=0.05$ ). This benefit of guidance-based training was not detected for the more skilled older participants. Similarly, we divided the younger group into less skilled participants $(n=14$, mean performance error $<0.09 \mathrm{~m}$ ), and more skilled young participants
( $n=16$ ). In the younger less skilled participants' group, the guidance-as-needed group showed better long-term learning (Fig. 6b, $P=0.05$ ), whereas this benefit was not detected for the more skilled younger participants.

Training with guidance improved learning of the steering task in curves, whereas it did not affect learning during straight lines

In a previous experiment (Marchal-Crespo and Reinkensmeyer 2008b), we hypothesized that the difference in learning between the guided and unguided groups arose because the physical guidance demonstrated the optimal moment at which to initiate turns to minimize the tracking error. If this hypothesis is correct, the guidance-as-needed group should have shown a significant reduction in tracking errors in parts of the circuit where turning too late or too early affected the tracking error the most. Thus, we broke the circuit into three different parts: the first half of the curves, the second half of the curves, and the straight lines between curves (see "Methods").

There was no significant difference in the error reduction during straight lines in both young and old participants 
between guidance groups (Fig. 7a, young participants $P=0.25$, old participants $P=0.24$ ). There was a significantly greater reduction in error for the first half of the curves for the guidance group compared with the no guidance groups for the younger participants (Fig. 7b, $P<0.003$ ), but not the older participants (Fig. 7b, $P=0.76$ ). In the young participants' group, there was a significant difference in the reduction of error created in the curves' second half among experimental groups (Fig. 7c, one-tailed $t$ test, $P=0.043$ ). Again, the difference did not hold for the older participants (Fig. 7c, $P=0.72$ ).

We also tested if there were differences between guidance groups in learning the turn-initiation distance before curves, and the turn rectification distance after curves. In contrast to our expectations, we found no significant difference in the change in turn-initiation distance between the guidance and no-guidance groups in both age groups, although old participants in the guidance-as-needed group tended to increase the turn-initiation distance when compared with old participants in the no assistance group (Fig. 7d, old participants $P=0.18$ ). There was a significant difference in the turnrectification distance reduction between groups in both age groups (Fig. 7e, young participants $P=0.016$, old participants $P=0.037$ ). In other words, participants who trained with guidance tended to rectify the trajectory after turns earlier when the guidance was removed.

We investigated how the turn-initiation distance and turnrectification distance (i.e. turn timing, see "Methods") are related to mean tracking error in each of the three circuit parts. There was a significant quadratic correlation between the turn-initiation distance and the mean tracking error during the curves' first part (young participants: $R^{2}=0.22$, $P<0.001$, old participants: $\left.R^{2}=0.25, P<0.001\right)$. There was a similar significant quadratic correlation between the turn-rectification distance and the mean tracking error during curves' second part (young participants: $R^{2}=0.28$,
Fig. 7 Long-term reduction in steering performance with and without haptic training. All plots compare the change in steering performance from trials 17 to 39 . Trial 17 was the last initial practice trial on Day 2 before training with guidance. Trial 39 was the final retention test trial on Day 3, 1 week later. a Error during straight line portions of the driving course. b Error during the first half of turns. c Error in second half of turns. a Mean increase in turninitiation distance. e Reduction in turn rectification distance
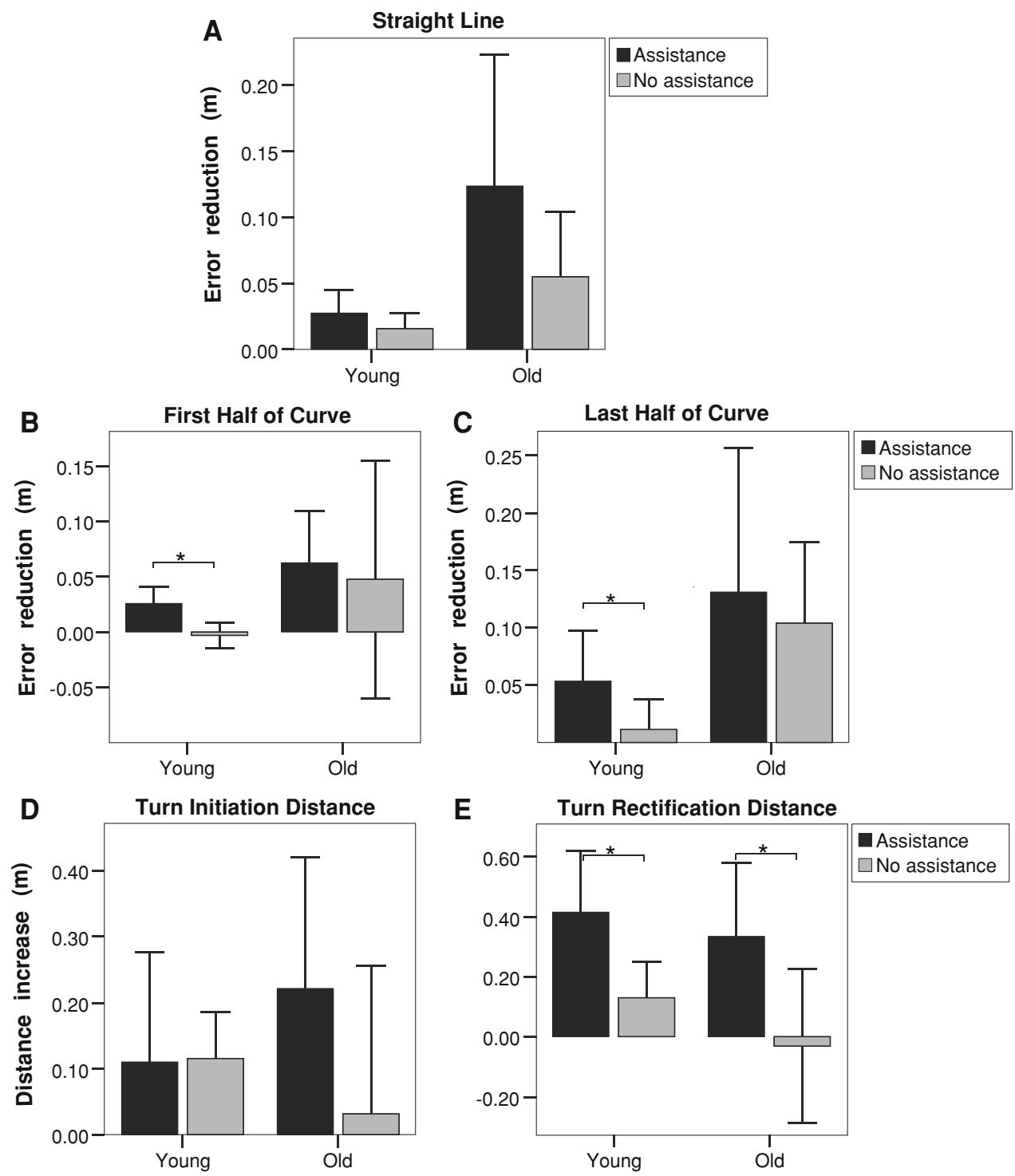
$P<0.001$, old participants: $\left.R^{2}=0.23, P<0.001\right)$. The mean tracking error created during straight lines was quadratically correlated with turn-initiation distance (young participants: $R^{2}=0.15, P<0.011$, old participants: $R^{2}=$ $0.39, P<0.001$ ), and with turn-rectification distance in the young subjects $\left(R^{2}=0.32, P<0.001\right)$. Thus, participants that learned better the optimal turn-initiation and turn-rectification distances indeed created smaller errors as they steered the simulated wheelchair through the simulated circuit.

Effect of age on driving performance and retention

Older participants not only systematically performed worse than younger subjects, but also apparently learned more slowly and forgot a greater percentage of the learned task during the 1-week layoffs between experimental sessions. The percentage error reduction was significantly smaller for the old participants' group on the first day (Fig. 3a, $P<0.022$ ), meaning that young subjects learned a larger percentage of the task during first day (i.e. they learned faster) than older individuals. Older participants experienced a greater increase in tracking error during the layoff from the end of day 1 (trial 15) to the beginning of day 2 (trial 16, 1 week later) when compared with younger subjects (Fig. 8a, one-tailed $t$ test, $P=0.045)$. However, when comparing the percentage of motor learning lost from last trial of day 1 and first trial on day 2, we found that retention was not significantly worse (Fig. 8 b, $P=0.26$ ).

We also looked at retention from days 2 to 3 (1 week later). Older participants had a significantly greater increase in error from the last trial on day 2 (trial 37) to trial 1 on day 3 (trial 38) (Fig. $8 \mathrm{c}, P=0.022$ ). Calculated as a percentage relative to the total error reduction from first trial on day 1 , and last trial on day 2, older participants showed significantly worse retention of skill from the day 2 testing session on day 3 (Fig. 8d, $P<0.045$ ). Specifically, participants in the young group showed a slight performance improvement (rather than loss of retention) from days 2 to 3 of $0.18 \pm 13.45 \%$, whereas old participants increased error by $23.45 \pm 43.54 \%$ after the 1-week layoff.

The addition of haptic guidance did not have any effect on retention from days 2 to 3 in any age group. We did not find any difference in performance loss (Mann-Whitney test, young $P=0.171$, old $P=0.861$ ), neither in percentage loss (Mann-Whitney test, young $P=0.206$, old $P=0.626)$.
Fig. 8 Retention of skill between testing sessions. a Mean tracking error increase from last trial on first day (trial 15), and first trial on second day (trial 16). b Percentage of learning loss in the first learning period. b Mean tracking error increase from last trial on second day (trial 37), and first trial on long-term retention on third day (trial 38). d Percentage of performance loss on Day 3 compared with Day 2
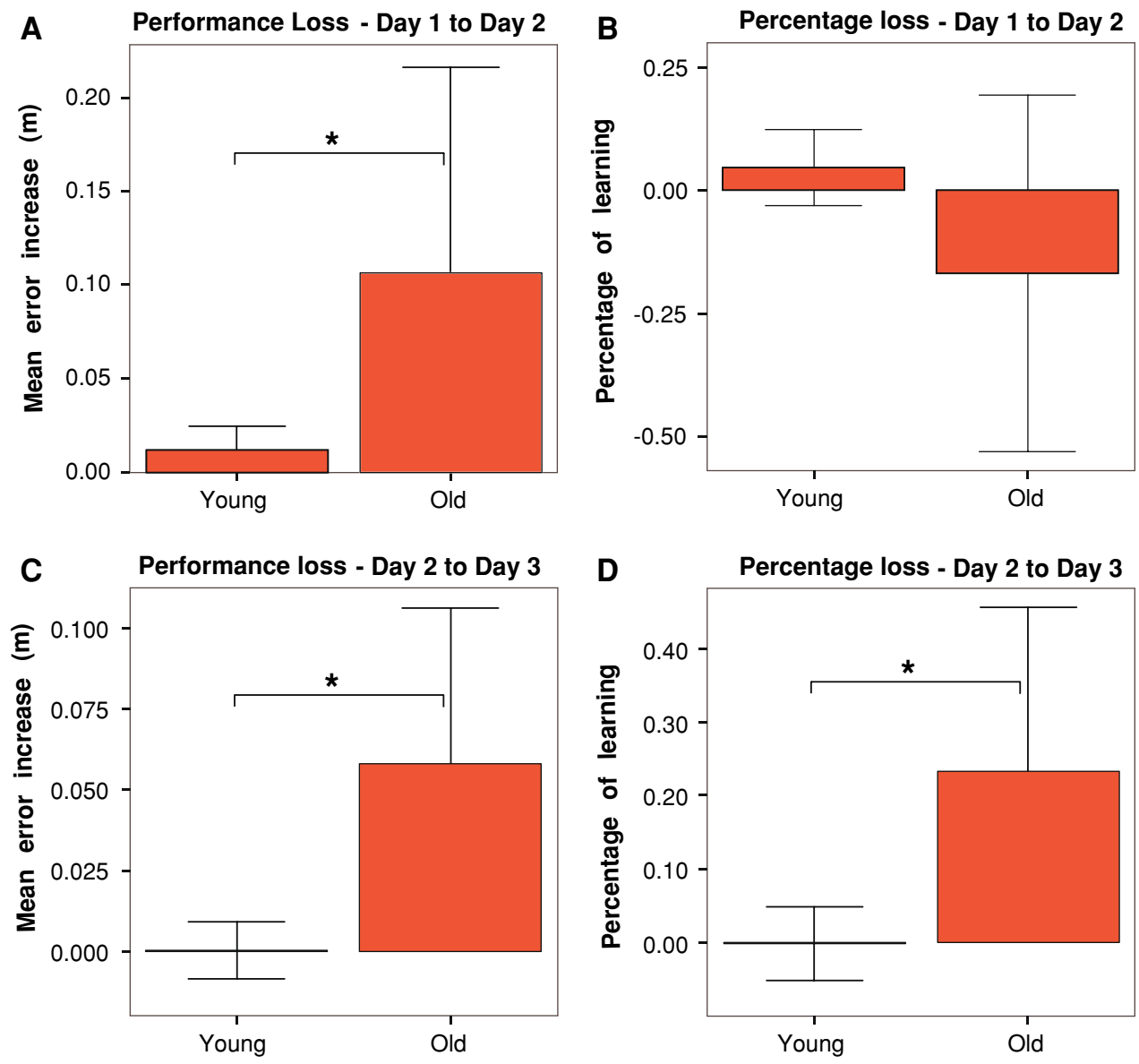


\section{Discussion}

We found that training with guidance-as-needed was more effective than training without guidance, for the task of learning to drive a new, simulated vehicle. For younger participants, training with guidance-as-needed produced a significant, long-term reduction in tracking error when compared with training without guidance. For older participants trained with guidance-as-needed, tracking error decreased, but the decrease was not significantly different from the error decrease when trained without guidance. Older participants taken as a group did however learn better to correct their course after turns, by initiating turns earlier, as a result of haptic training. In addition, for both less skilled younger and older participants, learning to drive with guidance-as-needed was significantly more effective than training without guidance. In discussing these results, we return to the three goals stated in the "Introduction" that this study was designed to address.

Can training with haptic guidance produce better long-term learning than unguided learning?

Training with haptic guidance-as-needed produced better long-term learning in certain steering performance measures than training without guidance (Fig. 5a). Guidanceas-needed was especially helpful in reducing errors created during curves (Fig. 7b, c), compared with performance during straight line portions of the track. Both young and old participants significantly reduced their turn-rectification distance (Fig. 7e) in response to haptic guidance. These results extend our previous findings (Marchal-Crespo and Reinkensmeyer 2008b), where we found that physical guidance enhanced short-term learning of the steering task. Note, however, that in the previous work we did not find a significant difference in the turn-rectification distance, although there was a non-significant tendency.

One possible interpretation of these results is that subjects performing complex tasks such as skiing (Wulf et al. 1998) and driving a vehicle learn to time their movement better with cues provided by haptic guidance, such as the moment to begin a turn when encountering a sharp curve or the moment to rectify after a curve (Marchal-Crespo and Reinkensmeyer 2008b). The concept that guidance can improve the learning of timing is also consistent with the results of Feygin et al. (2002), who showed a benefit of haptic guidance from a robot in learning to reproduce the temporal, but not spatial, characteristics of a complex spatiotemporal curve. The effect of haptic guidance on learning a visuo-manual tracking has been evaluated in recent studies, which found a positive effect of guidance on the time-related components of the dynamic task, such as an increase in speed and smoothness in tracking trajectories
(Bluteau et al. 2008), and a better learning of temporal patterns of force (Morris et al. 2007). In other words, there is emerging evidence that haptic guidance may be specifically useful for learning timing or force dynamics.

The form of guidance used, however, may affect the motor system's ability to benefit from guidance. We recently performed a pilot study of a simple task that requires precise timing (a pinball-like task), and found that while training with guidance allowed young adults to learn the trained task, it had no benefit compared with training without guidance (Marchal-Crespo and Reinkensmeyer 2008a). Subjects apparently reacted to guidance by "slacking" or relying on the form of guidance. Robotic guidance was applied on pseudo-random trials, and eliminated completely the performance errors on all guided trials. In the present study, guidance was applied continuously during a well-defined block of training trials, and the firmness of the guidance on these trials was gradually reduced over time, allowing increasing experience of error. No "slacking" effect was observed when guidance was removed, as the mean tracking error did not increase. This is not surprising, as the guidance had already been reduced by the adaptive guidance algorithm to a very small level by the time it was removed. The compliant, faded form of guidance used here may have ameliorated the negative effects of continuous guidance predicted by the Guidance Hypothesis. Thus, the principle that haptic guidance is specifically useful for learning timing may be conditioned on the provision of a schedule and form of guidance that allows the participant to gradually learn from the example of the guidance, instead of reacting to it as a perturbation.

The current results also suggest that guidance may only be helpful for specific sub-tasks of the steering task. Specifically, guidance benefited learning how to leave a curve, but not how to drive along a straight line. Driving along a straight line requires small responses proportional to the tracking errors, compared with the large, precisely timed movements required for turning into curves and straightening after turns. Research on motor adaption has emphasized that kinematic errors generated during movement are a fundamental neural signal that drives motor adaptation when steering the arm along a desired path (Fine and Thoroughman 2007; Halsband and Lange 2006; Krakauer 2006). We thus speculate that amplifying errors instead of reducing them when steering during straight lines might provide distinct benefits for this sub-task, improving driving performance by providing a more rich and varied experience of errors. If this speculation is correct, the implication is that optimal haptic training strategies will necessarily incorporate different forms of assistance or error amplification based on the specific nature of the current sub-task being practiced. Examples of research towards the study of optimal forms of haptic 
training strategies to enhance motor learning can be found in Patton et al. (2006) and Bayart et al. (2005).

Generalization is an important component of learning. The task we studied was fairly complex, with curves to the right, to the left and straight lines, so it required learning a relatively broad range of driving behaviors. However, we did not vary the wheelchair speed during training or testing, or the driving course layout. A logical next step for future studies would be to examine to what extent training with haptic guidance generalizes to novel driving conditions.

Finally, based on the timing improvement results, it could be argued that the effect of haptic guidance contributed to the development of a cognitive skill, rather than a motor program. Indeed, steering well requires a realization that turns must begin early, and straightening the vehicle when it came out a turn required visual-spatial processing of a virtual reality scene that had been skewed by the turn. However, the cognitive skills were likely internalized at the beginning of the training session, when subjects learned the cause-effect sequence that a late turn resulted in a large error. The optimal time required to start the turn movement before curves, however, is a critical element in motor learning (Schmidt and Lee 2005). We argue that haptic guidance contributed to acquire the correct time to start the turning movement, thus, a critical variable in motor programs, while the cognitive skills were internalized without the need of the haptic guidance during first training trials.

Are the training benefits of haptic guidance age dependent?

A decline of motor performance with age has been observed in many previous studies with a variety of movement tasks (Voelcker-Rehage 2008; Voelcker-Rehage and Willim-czik 2006; Etnier 1998; Tunney et al. 1998). Studies have also found that aging slows the rate of motor learning (Swinnen 1998; Brosseau et al. 2007; Wishart et al. 2002). The observed learning deficits in old participants have been hypothesized to be a consequence of a decreased capability to overcome "over-learned" coordination patterns to a specific task, i.e. old subjects have difficulties to learn variations on a task they are already skilled at Swinnen (1998) and Brosseau et al. (2007).

In the present study, older participants performed systematically worse than younger participants throughout all stages of the task, and had poorer retention of the learned driving skill following the week rest period. However, we did not find significant differences between age groups in the percentage of error reduction gained following training with haptic guidance (Fig. 5b). This was despite the fact that the driving task studied here can be characterized very well as a variation of an over-learned task. Thus, the over- learned phenomenon does not appear to limit learning from haptic guidance by the elderly.

Are the training benefits of haptic guidance skill dependent?

Haptic guidance was found to be more beneficial for less skilled participants in the current study. A possible explanation for this result can be found in the challenge point theory proposed by Guadagnoli et al. (2004). The challenge point hypothesis states that optimal learning is achieved when the difficulty of the task is appropriate for the individual participant's level of expertise (i.e. when the challenge point is reached). Thus, providing a difficult task to a less skilled participant would be predicted to result in less learning with a similar amount of practice, as compared to training when the task is adjusted to be at an appropriate skill level. Likewise, providing an easy task to a proficient participant would not be predicted to promote learning, as little new information is delivered and new skills are not mastered. In the present experiment, reducing the task's difficulty through haptic guidance may have benefited less skilled participants more because it made the task appropriately challenging. Guidance may not have benefited more skilled participants, since for them it made the task require little effort or attention.

The finding that the haptic guidance-as-needed strategy seems to enhance motor learning based on participants' initial skill level suggests that care should be taken to design individualized haptic training algorithms based on the participant's level of performance. These results are consistent with a recent preliminary study in the rehabilitation literature that showed that more impaired stroke patients benefited more from haptic guidance training during the practice of a reaching task, whereas error amplification was more beneficial for the least impaired patients (Cesqui et al. 2008). Matching the haptic training strategy to the trainee's skill level and the motor task properties may provide the greatest opportunity for learning.

Acknowledgment We would like to thank Crystal Hessler for assisting with data collection. This work was supported in part by NIH N01-HD-3-3352 from NCMRR and NIBIB.

Open Access This article is distributed under the terms of the Creative Commons Attribution Noncommercial License which permits any noncommercial use, distribution, and reproduction in any medium, provided the original author(s) and source are credited.

\section{References}

Armstrong $T$ (1970) Training for the production of memorized movement patterns. Technical report 26, University of Michigan, Human Performance Center, Ann Arbor 
Bayart B, Pocheville A, Kheddar A (2005) An adaptive haptic guidance software module for i-touch: example through a handwriting teaching simulation and a $3 \mathrm{~d}$ maze. In: IEEE international workshop on haptic audio visual environments and their applications

Bluteau J, Coquillart S, Payan Y, Gentaz E (2008) Haptic guidance improves the visuo-manual tracking of trajectories. PLoS ONE 3(3): e1775

Brosseau J, Potvin M-J, Rouleau I (2007) Aging affects motor skill learning when the task requires inhibitory control. Dev Neuropsychol 32(1):597-613

Carnahan H, Vandervoort A, Swanson L (1996) The influence of summary knowledge of results and aging on motor learning. Res Q Exerc Sport 67:280-287

Cesqui B, Aliboni S, Mazzoleni S, Carrozza M, Posteraro F, Micera S (2008) On the use of divergent force fields in robot-mediated neurorehabilitation. Biomedical Robotics and Biomechatronics, 2008. BioRob 2008. 2nd IEEE RAS \& EMBS international conference on, pp 854-861

Dijk H, van Mulder T, Hermens HJ (2007) Effects of age and content of augmented feedback on learning an isometric force-production task. Exp Aging Res 33(3):341-353

Emken J, Benitez R, Reinkensmeyer D (2007) Human-robot cooperative movement training: learning a novel sensory motor transformation during walking with robotic assistance-asneeded. J NeuroEng Rehabil 4(8)

Etnier JL (1998) Motor performance and motor learning as a function of age and fitness. Res Q Exerc Sport 32(1):597-613

Feygin D, Keehner M, Tendick R (2002) Haptic guidance: experimental evaluation of a haptic training method for a perceptual motor skill. In: Proceedings of the 10th symposium on haptic interfaces for virtual environment and teleoperator systems. (HAPTICS.02), pp 40-47

Fine MS, Thoroughman KA (2007) Trial-by-trial transformation of error into sensorimotor adaptation changes with environmental dynamics. J Neurophysiol 98(3):1392-1404

Gillespie R, O'Modhrain M, Tang P, Zaretzky D, Pham C (1998) The virtual teacher. In: Proceedings of the ASME dynamic systems and control division. Anaheim, CA, pp 171-178

Griffths PG, Gillespie RB (2005) Sharing control between humans and automation using haptic interface: primary and secondary task performance benefits. Hum Factors: J Hum Factors Ergonom Soc 47(3):574-590

Guadagnoli M, Lee T (2004) Challenge point: a framework for conceptualizing the effects of various practice conditions in motor learning. J Motor Behav 36(2):212-224

Hagman JD (1983) Presentation- and test-trial effects on acquisition and retention of distance and location. J Exp Psychol Lear Mem Cogn 9(2):334-345

Halsband U, Lange R (2006) Motor learning in man: a review of functional and clinical studies. J Physiol Paris 99(4-6): 414-424

Hedel H, Dietz V (2004) The influence of age on learning a locomotor task. Clin Neurophysiol 115:2134-2143

Jones LA, Sarter NB (2008) Tactile displays: guidance for their design and application. Hum Factors: J Hum Factors Ergonom Soc 50(1):90-111

Kahn L, Zygman M, Rymer W, Reinkensmeyer D (2006) Robotassisted reaching exercise promotes arm movement recovery in chronic hemiparetic stroke: a randomized controlled pilot study. J NeuroEngineering Rehabil 3(12)

Krakauer JW (2006) Motor learning: its relevance to stroke recovery and neurorehabilitation. Curr Opin Neurol 19(1):84-90

Liu J, Cramer S, Reinkensmeyer D (2006) Learning to perform a new movement with robotic assistance: comparison of haptic guidance and visual demonstration. J NeuroEng Rehabil 3(1):495506
Marchal-Crespo L, Reinkensmeyer DJ (2008a) Effect of robotic guidance on motor learning of a timing task. 2nd IEEE RAS and EMBS international conference on biomedical robotics and biomechatronics, pp 199-204

Marchal-Crespo L, Reinkensmeyer DJ (2008b) Haptic guidance can enhance motor learning of a steering tasks. J Motor Behav 40(6):545-557

Morris D, Tan H, Barbagli F, Chang T, Salisbury K (2007) Haptic feedback enhances force skill learning. In: Whc '07: Proceedings of the second joint euro-haptics conference and symposium on haptic interfaces for virtual environment and teleoperator systems, pp 21-26

O'Malley M, Gupta A, Gen M, Li Y (2006) Shared control in haptic systems for performance enhancement and training. J Dyn Syst Meas Control 128:75-85

Patton JL, Stoykov ME, Kovic M, Mussa-Ivaldi FA (2006) Evaluation of robotic training forces that either enhance or reduce error in chronic hemiparetic stroke survivors. Exp Brain Res 168(3):368-383

Salmoni A, Schmidt R, Walter C (1984) Knowledge of results and motor learning: a review and critical reappraisal. Psychol Bull 95(3):355-386

Schmidt R, Bjork R (1992) New conceptualizations of practice: common principles in three paradigms suggest new concepts for training. Psychol Sci 3(4):207-217

Schmidt R, Lee T (2005). Motor control and learning: a behavioral emphasis. Human Kinetics Publishers, Champaign

Suzuki K, Jansson H (2003) An analysis of driver's steering behaviour during auditory or haptic warnings for the designing of lane departure warning system. JSAE Rev 24(1):65-70

Swanson L, Lee T (1992) Effects of aging and schedules of knowledge of results on motor learning. J Gerontol B Psychol Sci 47:406-411

Swinnen SP (1998) Age-related deficits in motor learning and differences in feedback processing during the production of a bimanual coordination pattern. Cogn Neuropsychol 15(5):439-466

Tsutsui S, Imanaka K (2003) Effect of manual guidance on acquiring a new bimanual coordination pattern. Res Q Exerc Sport 74(1):104-109

Tunney N, Taylor LF, Gaddy M, Marcoux A, Pearce N, Tamanini J, et al. (1998). Aging and motor learning of a functional motor. Phys Occup Ther Geriatr Curr Trends Geriatr Rehabil 21(3):1-16

Voelcker-Rehage C (2008) Motor-skill learning in older adults: a review of studies on age-related differences. Eur Rev Aging Phys Activity 5:5-16

Voelcker-Rehage C, Willimczik K (2006) Motor plasticity in a juggling task in older adultsa developmental study. Age Ageing 35:422-427

Wallis R, Koh M (2002) The effect of a fast bowling harness in cricket: an intervention study. J Sports Sci 20:495-506

Winstein C, Pohl PS, Lewthwaite R (1994) Effects of physical guidance and knowledge of results on motor learning: support for the guidance hypothesis. Res Q Exerc Sport 65(4):316-323

Wishart L, Lee $\mathrm{T}$ (1997) Effects of aging and reduced relative frequency of knowledge of results on learning a motor skill. Percept Mot Skills 84:1107-1122

Wishart L, Lee T, Cunningham S, Murdoch J (2002) Age-related differences and the role of augmented visual feedback in learning a bimanual coordination pattern. Acta Psychol 110(23):247-263

Wulf G, Shea CH, Whitacre C (1998) Physical-guidance benefits in learning a complex motor skill. J Motor Behav 30(4): 367-380

Zar J (1984) Biostatistical analysis. Prentice-Hall, Englewood Cliffs

Zhai S, Accot J, Woltjer R (2004) Human action laws in electronic virtual worlds: an empirical study of path steering performance in vr. Presence: Teleoper Virtual Environ 13(2):113-127 